\title{
Solving Linear Programming Problems with Fuzzy Data
}

\author{
Michael Gr. Voskoglou \\ Department of Mathematical Sciences, School of Technological Applications \\ Graduate Technological Educational Institute of Western Greece \\ Email: mvosk@hol.gr; voskoglou@teiwest.gr
}

\begin{abstract}
A Fuzzy Linear Programming problem differs from an ordinary one to the fact that the coefficients of its objective function and / or the technological coefficients and constants of its constraints are fuzzy instead of real numbers. In this work a new method is developed for solving such kind of problems by ranking the fuzzy numbers involved and by solving the obtained in this way ordinary Linear Programming problem with the standard theory. The values of the decision variables may then be converted to fuzzy numbers in order to facilitate a fuzzy expression of the problem's optimal solution, but this must be strictly checked to avoid non-creditable expressions. Examples involving triangular and trapezoidal fuzzy numbers are also presented in the paper illustrating the applicability of our method to real-life applications.
\end{abstract}

Keywords: linear programming (LP), SIMPLEX method, duality, fuzzy numbers (FNs), triangular FNs (TFNs), trapezoidal FNs (TpFNs), centre of gravity (COG) defuzzification technique, ranking of FNs, degree of fuzziness (DoF), fuzzy LP

\section{Introduction}

It is well known that Linear Programming $(L P)$ is a technique for the optimization (maximization or minimization) of a linear objective function subject to linear equality and inequality constraints. The feasible region of a LP problem is a convex polytope, which is a generalization of the three-dimensional polyhedron in the $n$-dimensional real space $\boldsymbol{R}^{n}$, where $n$ is an integer, $n \geq 2$.

An LP algorithm determines a point of the LP polytope, where the objective function takes its optimal value, if such a point exists. In 1947 George B. Dantzic invented the SIMPLEX algorithm [1] that for the first time efficiently tackled the LP problem in most cases. Further, in 1948 Dantzic, adopting a conjecture of John von Neuman, who worked on an equivalent problem in Game Theory, provided a formal proof of the theory of Duality [2]. According to the above theory every LP problem has a dual problem the optimal solution of which, if there exists, provides an optimal solution of the original problem. For general facts about the SIMPLEX algorithm we refer to Chapters 3 and 4 of [3].

LP, apart from mathematics, is widely used nowadays in business and economics, in several engineering problems, etc. Many practical problems of Operations Research can be expressed as LP problems. However, in large and complex systems, like the socio-economic, the biological ones, etc. ., it is often very difficult to solve the LP problems with the standard theory satisfactorily, since the necessary data cannot be easily determined precisely and therefore estimates of them are used in practice. The reason for this is that such kind of systems usually involves many different and constantly changing factors, the relationships among which are indeterminate, making their operation mechanisms not clear. In order to obtain good results in such cases one may apply either techniques of fuzzy logic (Fuzzy LP, e.g. see [4-6], etc.) or of the grey systems theory (Grey $L P$, e.g. see [7, 8], etc.).

In this work we develop a new technique for solving fuzzy LP problems. The rest of the paper is formulated as follows: In the second Section the background information is recalled about Fuzzy Numbers $(F N s)$ which is necessary for the understanding of the paper. In the third Section our method for solving fuzzy LP problems is developed and examples are presented illustrating it. Finally, the fourth and last Section contains our conclusion and some suggestions for future research on the subject. 


\section{$2 \quad$ Fuzzy Numbers}

\subsection{Introductory Concepts}

FL, due to its nature of characterizing the ambiguous real life situations with multiple values, offers rich resources for handling problems with approximate data. This multiple-valued logic, being an extension / complement of the classical bi-valued Logic of Aristotle, is based on the notion of Fuzzy Set (FS), introduced by Zadeh in 1965 [9] as follows:

Definition 1: A FS on the universal set $U$ of the discourse (or a fuzzy subset of $U$ ) is a set of ordered pairs of the form $A=\left\{\left(x, m_{A}(x)\right): x \in U\right\}$, defined in terms of a membership function $m_{A}: U \rightarrow[0,1]$ that assigns to each element of $U$ a real value from the interval $[0,1]$.

The value $m_{A}(x)$ is called the membership degree of $x$ in $A$. The greater is $m_{A}(x)$, the better $x$ satisfies the characteristic property of $A$. The choice of the membership function is not unique depending on the user's subjective goals and it is usually based on statistical or empirical observations. However, a necessary condition for a FS to provide a reliable description of the corresponding real situation is that its membership function's definition satisfies the common sense. Note that, for reasons of simplicity, many authors identify a FS with its membership function.

A crisp subset $A$ of $U$ can be considered as a FS on $U$ with $m_{A}(x)=1$, if $x \in A$ and $m_{A}(x)=0$, if $x \notin A$. In this way most properties and operations of crisp sets can be extended to corresponding properties and operations of FS. For general facts on FS we refer to the book [10].

FNs play an important role in fuzzy mathematics analogous to the role played by the ordinary numbers in crisp mathematics. The general definition of a FN is the following:

Definition 2: An FN is an FS $A$ on the set $\boldsymbol{R}$ of real numbers with membership function $m_{A}: \boldsymbol{R} \rightarrow[0$, 1], such that:

- $A$ is normal, i.e. there exists $x$ in $\boldsymbol{R}$ such that $m_{A}(x)=1$.

- $A$ is convex, i.e. all its $a$-cuts $\mathrm{A}^{a}=\left\{x \in \mathrm{U}: m_{A}(\mathrm{x}) \geq a\right\}$, with $a$ in $[0,1]$, are closed real intervals.

- Its membership function $y=m_{A}(x)$ is a piecewise continuous function.

Note that one can define with two different, but equivalent to each other methods, the basic arithmetic operations on FNs. as we do for the ordinary numbers [11]. However, since both methods involve laborious calculations in the general case, in practical applications it is usually preferred to utilize special forms of FNs, for which these operations can be performed easily.

For general facts on FNs we refer to the book [11].

\subsection{Triangular and Trapezoidal Fuzzy Numbers}

In this work we are going to use the two simpler forms of FNs, the Triangular FNs (TFNs) and the Trapezoidal FNs (TpFNs).

Definition 3: Let $\alpha, b$ and $c$ be real numbers with $\alpha<b<c$. Then the TFN $A=(\alpha, b, c)$ is an FN with membership function:

$$
y=m(x)=\left\{\begin{array}{cl}
\frac{x-a}{b-a}, & x \in[a, b] \\
\frac{c-x}{c-b}, & x \in[b, c] \\
0, & x<a, x>c
\end{array}\right.
$$

The membership function's graph of the TFN $(\alpha, b, c)$ and its Centre of Gravity (COG) are presented in Fig. 1. 


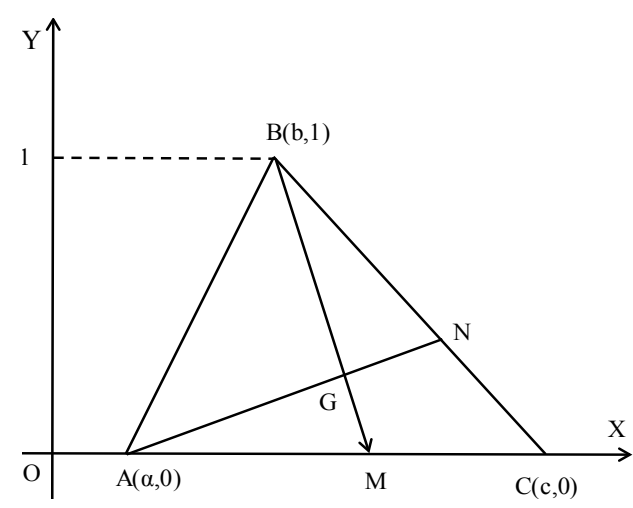

Figure 1. Graph and COG of the TFN $(\alpha, b, c)$

It can be shown [11] that the two general methods for performing operations on FNs lead to the following simple rules for the addition and subtraction of TFNs:

Proposition 1: Let $A=\left(a_{1}, a_{2}, a_{3}\right)$ and $B=\left(b_{1}, b_{2}, b_{3}\right)$ be two TFNs. Then:

- The sum of them is the TFN $A+B=\left(a_{1}+b_{1}, a_{2}+b_{2}, a_{3}+b_{3}\right)$.

- The difference of them is the TFN $A-B=\left(a_{1}-b_{3}, a_{2}-b_{2}, a_{3}-b_{1}\right)$.

On the contrary, the product $A . B$ and the quotient $A: B$ are FNs which are not TFNs, apart from some special cases. For example, if all the entries of $A$ and $B$ are positive numbers, then one can approximately write that $A . B=\left(a_{1} b_{1}, a_{2} b_{2}, a_{3} b_{3}\right)$ and $A: B=\left(a_{1} / b_{3}, a_{2} / b_{2}, a_{3} / b_{1}\right)$.

Definition 4: Let $\alpha<b \leq c<d$ be real numbers. Then the $\operatorname{TpFN}(\alpha, b, c, d)$ is a FN with membership function:

$$
y=m(x)=\left\{\begin{array}{lc}
\frac{x-a}{b-a} & , \quad x \in[a, b] \\
x=1, & , \quad x \in[b, c] \\
\frac{d-x}{d-c}, & x \in[c, d] \\
0, & x<a \text { and } x>d
\end{array}\right.
$$

The graph of the $\operatorname{TpFN}(a, b, c, d)$ and the COGs of its parts (two triangles and a rectangle) are shown in Fig. 2

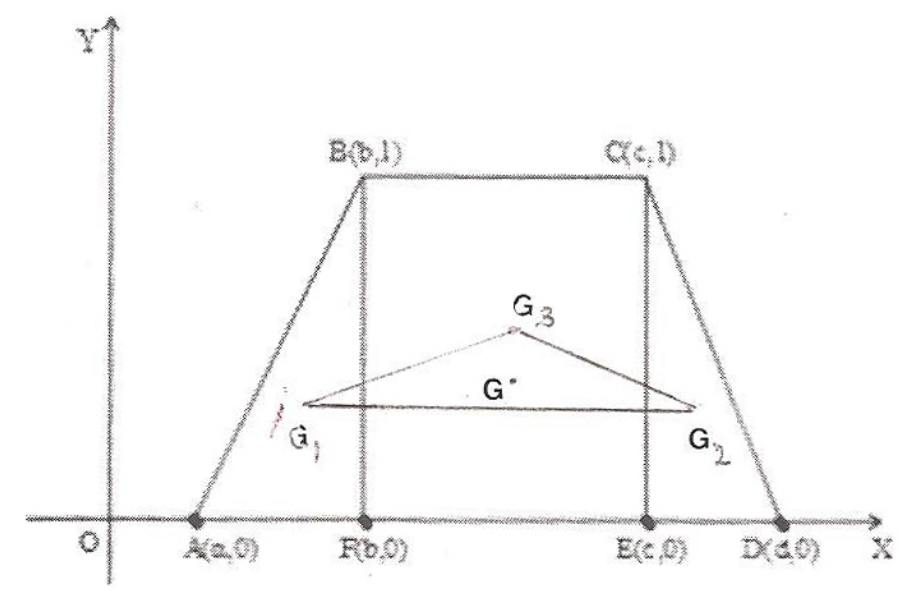

Figure 2. Graph of the TpFN $(\alpha, b, c, d)$ and the COGs of its parts

It is easy to observe that the $\operatorname{TFN}(a, b, d)$ is a special case of the $\operatorname{TpFN}(a, b, c, d)$ with $c=b$; in other words the TpFNs are generalizations of the TFNs.

Similarly with the TFNs it can be shown [11] that, if $A=\left(a_{1}, a_{2}, a_{3}, a_{4}\right)$ and $B=\left(b_{1}, b_{2}, b_{3}, b_{4}\right)$ are given TpFNs, then 
and.

$$
A+B=\left(a_{1}+b_{1}, a_{2}+b_{2}, a_{3}+b_{3}, a_{4}+b_{4}\right)
$$

$$
\mathrm{A}-\mathrm{B}=\left(a_{1}-b_{4}, a_{2}-b_{3}, a_{3}-b_{2}, a_{3}-b_{4}\right),
$$

whereas $A$. $B$ and $A: B$ are not TpFNs in general.

Remark 1: The TFNs and the TpFNs are special cases of the $L R-F N s$ of Dubois and Prade [12]. Generalizing the definitions of TFNs and TpFNs one can define $n$-agonal FNs of the form $\left(a_{1}, a_{2}, \ldots, a_{n}\right)$ for any integer $n, n \geq 3$ (e.g. see Section 2 of [13] for the definition of the hexagonal FNs).

For the needs of the present work we also introduce the following definition: 0

Definition 5: The Degree of Fuzziness (DoF) of the n-agonal FN $A=\left(a_{1}, \ldots, a_{n}\right)$ is defined to be the real number $\mathrm{D}=a_{n}-a_{1}$. We write then $\operatorname{DoF}(A)=\mathrm{D}$.

\subsection{Defuzzification and Ranking of TFNs and TpFNs}

The general approach for solving a problem using principles of FL involves the following steps:

- Fuzzification of the problem's data by representing them with properly defined FSs.

- Evaluation of the fuzzy data by applying principles and methods of FL in order to find the problem's solution in the form of a unique FS.

- Defuzzification of the problem's solution in order to "translate" it into the natural language for applying it to the original real-life problem.

The most popular defuzzification method is perhaps the Centre of Gravity (COG) technique, according to which the problem's fuzzy outcomes are represented by the coordinates of the COG of the membership's function graph of the FS representing its solution [14]. The following two propositions concern the defuzzification of a given TFN and TpFN respectively with the COG technique:

Proposition 2: The coordinates $(X, Y)$ of the COG of the graph of the TFN $(\alpha, b, c)$ are calculated by the formulas

$$
X=\frac{a+b+c}{3}, Y=\frac{1}{3}
$$

Proof: The graph of the TFN $(\alpha, b, c)$ is the triangle ABC of Figure 1, with A $(\alpha, 0), \mathrm{B}(b, 1)$ and $\mathrm{C}(c$, $0)$. Then, the COG, say G, of ABC is the intersection point of its medians $\mathrm{AN}$ and BM, where $\mathrm{N}\left(\frac{b+c}{2}\right.$, $\left.\frac{1}{2}\right)$ and $\mathrm{M}\left(\frac{a+c}{2}, 0\right)$. Therefore, it is a routine process of Analytic Geometry to form the equations of the straight lines defined by the line segments $\mathrm{AN}$ and $\mathrm{BM}$ and then to determine the coordinates of G by solving the linear system of those two equations.

Proposition 3: Consider the graph of the $\operatorname{TpFN}(\alpha, b, c, d)$ (Figure 2). Let $\mathrm{G}_{1}$ and $\mathrm{G}_{2}$ be the COGs of the rectangular triangles $\mathrm{AEB}$ and $\mathrm{CFD}$ and let $\mathrm{G}_{3}$ be the $\mathrm{COG}$ of the rectangle $\mathrm{BEFC}$ respectively. Then $\mathrm{G}_{1} \mathrm{G}_{2} \mathrm{G}_{3}$ is always a triangle, whose $\mathrm{COG}$ has coordinates

$$
X=\frac{2(a+d)+7(b+c)}{18}, Y=\frac{7}{18} .
$$

Proof: By Proposition 2 one finds that $\mathrm{G}_{1}\left(\frac{a+2 b}{3}, \frac{1}{3}\right)$ and $\mathrm{G}_{2}\left(\frac{d+2 c}{3}, \frac{1}{3}\right)$. Further, it is easy to check that the GOG $\mathrm{G}_{3}$ of the rectangle BCFD, being the intersection of its diagonals, has coordinates $\left(\frac{b+c}{2}, \frac{1}{2}\right)$. The $y$ - coordinates of all points of the straight line defined by the line segment $G_{1} G_{2}$ are equal to $1 / 3$, therefore the point $\mathrm{G}_{3}$, having $\mathrm{y}$ - coordinate equal to $1 / 2$, does not belong to this line. Hence, by Proposition 2, the COG $\mathrm{G}^{\prime}$ of the triangle $\mathrm{G}_{1} \mathrm{G}_{2} \mathrm{G}_{3}$ has coordinates

$$
X=\left(\frac{a+2 b}{3}+\frac{d+2 c}{3}+\frac{b+c}{2}\right): 3=\frac{2(a+d)+7(b+c)}{18}
$$

and 


$$
Y=\left(\frac{1}{3}+\frac{1}{3}+\frac{1}{2}\right): 3=\frac{7}{18}
$$

Remark 2: Since the COGs $\mathrm{G}_{1}, \mathrm{G}_{2}$ and $\mathrm{G}_{3}$ are the balancing points of the triangles AEB and CFD and of the rectangle BEFC respectively, the COG $\mathrm{G}^{\prime}$ of the triangle $\mathrm{G}_{1} \mathrm{G}_{2} \mathrm{G}_{3}$, being the balancing point of the triangle formed by those COGs, may be considered instead of the COG G of the trapezoid ABCD as the tool for defuzzifying the $\operatorname{TpFN}(a, b, c, d)$. An advantage of the choice of $\mathrm{G}^{\prime}$ is that the formulas calculating the coordinates of it are simpler than those calculating the COG of the trapezoid ABCD (Proposition 11, Chapter 7 of [15]).

An important problem of the fuzzy arithmetic is the ordering of FNs, i.e. the process of determining whether a given FN is larger or smaller than another one. This problem can be solved through the introduction of a ranking function, say R, which maps each FN on the real line, where a natural order exists. Several ranking methods have been proposed until today, like the lexicographic screening [16], the use of an area between the Centroid and original points [17], the subinterval average method [13], etc.

Here, under the light of Propositions 2 and 3 respectively, we define the ranking functions for a TFN and a TpFN as follows:

Definition 6: Let $A$ be a FN. Then:

i) If $A\{\alpha, b, c)$ is a TFN, we define $\mathrm{R}(A)=\frac{a+b+c}{3}$.

ii) If $A\{\alpha, b, c, d)$ is a TpFN, we define $\mathrm{R}(A)=\frac{2(a+d)+7(b+c)}{18}$.

Proposition 4: Let $A$ be a TFN with $\operatorname{DoF}(A)=\mathrm{D}$ and $\mathrm{R}(A)=\mathrm{R}$. Then $A$ can be written in the form $A=(\alpha, 3 \mathrm{R}-2 \alpha-\mathrm{D}, \alpha+\mathrm{D})$

where $\alpha$ is a real number such that

$$
\mathrm{R}-\frac{2 \mathrm{D}}{3}<\alpha<\mathrm{R}-\frac{\mathrm{D}}{3} .
$$

Proof: Let $A(\alpha, b, c)$ be the given TFN, where $\alpha, b, c$ are real numbers such that $\alpha<b<c$. Then, since $\mathrm{D}(A)=c-\alpha=\mathrm{D}$, it is $c=\alpha+\mathrm{D}$. Therefore,

$$
\mathrm{R}(A)=\frac{a+b+c}{3}=\frac{2 a+b+\mathrm{D}}{3}=\mathrm{R}
$$

which gives that $b=3 \mathrm{R}-2 \alpha-\mathrm{D}$.

Consequently we have that $\alpha<3 \mathrm{R}-2 \alpha-\mathrm{D}<\alpha+\mathrm{D}$. The left side of the last inequality implies that $3 \alpha<$ $3 \mathrm{R}-\mathrm{D}$, or $\alpha<\mathrm{R}-\frac{\mathrm{D}}{3}$. Also its right side implies that $-3 \alpha<2 \mathrm{D}-3 \mathrm{R}$, or $\alpha>\mathrm{R}-\frac{2 \mathrm{D}}{3}$ and this completes the proof.

The corresponding situation is more complicated if $A$ is a TpFN. In this case we have:

Proposition 5: Let $A$ be a TpFN with $\operatorname{DoF}(A)=\mathrm{D}$ and $\mathrm{R}(A)=\mathrm{R}$. Then $A$ can be written in the form $A=(\alpha, \mathrm{b}, \mathrm{c}, \alpha+\mathrm{D})$, where $\alpha, b$ and $c$ are real numbers such that

$$
\alpha<b \leq c<a+\mathrm{D}, b+c=\frac{18 \mathrm{R}-4 a-2 \mathrm{D}}{7} .
$$

Proof: Let $A(\alpha, b, c, d)$ be the given TFN, with $\alpha, b, c, d$ real numbers such that $a<b \leq c<d$. Since $\mathrm{D}(A)=d-\alpha=\mathrm{D}$, it is $d=\alpha+\mathrm{D}$. Also, by Definition 6(ii) we have that

$$
\mathrm{R}=\frac{2(2 a+D)+7(b+c)}{18}
$$

wherefrom one gets the expression of $b+c$ in the required form.

\section{A Method of Solving Fuzzy LP Problems}

The general form of a Fuzzy LP problem is the following:

Maximize (or minimize) the linear expression

$$
\mathrm{F}=\mathrm{A}_{1} \mathrm{x}_{1}+\mathrm{A}_{2} \mathrm{x}_{2}+\ldots .+\mathrm{A}_{\mathrm{n}} \mathrm{x}_{\mathrm{n}}
$$


subject to constraints of the form

$$
x_{j} \geq 0, A_{i 1} x_{1}+A_{i 2} x_{2}+\ldots . .+A_{i n} x_{n} \leq(\geq) B_{i}
$$

where $\mathrm{i}=1,2, \ldots, \mathrm{m}, \mathrm{j}=1,2, \ldots, \mathrm{n}$ and $\mathrm{A}_{\mathrm{j}}, \mathrm{A}_{\mathrm{ij}}, \mathrm{B}_{\mathrm{i}}$ are FNs.

The proposed in this work method for solving a Fuzzy LP problem involves the following steps:

- Ranking of the FNs $A_{j}, A_{i j}$ and $B_{i}$.

- Solution of the obtained in the previous step ordinary LP problem with the standard theory.

- Conversion of the values of the decision variables in the optimal solution to FNs with the desired DoF.

The last step is not compulsory, but it is useful in problems of vague structure, where a fuzzy expression of their solution is often preferable than the crisp one.

The following examples illustrate the applicability of our method: to real life applications:

Example 1: A furniture-making factory constructs tables and desks. It has been statistically estimated that the construction of a group of tables needs 2 - 3 working hours (w.h.) for assembling, $2.5-3.5$ w.h. for elaboration (plane, etc.) and $0.75-1.25$ w.h. for polishing. On the other hand, the construction of a group of desks needs $0.8-1.2,2-4$ and 1.5 - 2.5 w.h. for each of the above procedures respectively. According to the factory's existing number of workers, no more than $20 \mathrm{w} . \mathrm{h}$. per day can be spent for the assembling, no more than $30 \mathrm{w} . \mathrm{h}$. for the elaboration and no more than 18 w.h. for the polishing of the tables and desks. If the profit from the sale of a group of tables is between 2.7 and 3.3 thousand euros and of a group of desks between 3.8 and 4.2 thousand euros, ${ }^{1}$ find how many groups of tables and desks should be constructed daily to maximize the factory's total profit. Express the problem's optimal solution with TFNs of DoF equal to 1.

Solution: Let $\mathrm{x}_{1}$ and $\mathrm{x}_{2}$ be the groups of tables and desks to be constructed daily. Then, using TFNs, the problem can be mathematically formulated as follows: ${ }^{2}$

Maximize $\mathrm{F}=(2.7,3,3.3) \mathrm{x}_{1}+(3.8,4,4.2) \mathrm{x}_{2}$ subject to constraints $\mathrm{x}_{1}, \mathrm{x}_{2} \geq 0$ and

$$
\begin{gathered}
(2,2.5,3) \mathrm{x}_{1}+(0.8,1,1.2] \mathrm{x}_{2} \leq(19,20,21) \\
(2.5,3,3.5) \mathrm{x}_{1}+(2,3,4) \mathrm{x}_{2} \leq(29,30,31)
\end{gathered}
$$$$
(0.75,1,1.25) \mathrm{x}_{1}+(1.5,2,2.5) \mathrm{x}_{2} \leq(15,16,17)
$$

The ranking of the TFNs involved leads to the following LP maximization problem of the canonical form: Maximize $\mathrm{f}\left(\mathrm{x}_{1}, \mathrm{x}_{2}\right)=3 \mathrm{x}_{1}+4 \mathrm{x}_{2}$ subject to the constraints $\mathrm{x}_{1}, \mathrm{x}_{2} \geq 0$ and

$$
\begin{gathered}
2.5 \mathrm{x}_{1}+\mathrm{x}_{2} \leq 20 \\
3 \mathrm{x}_{1}+3 \mathrm{x}_{2} \leq 30 \\
\mathrm{x}_{1}+2 \mathrm{x}_{2} \leq 16
\end{gathered}
$$

Adding the slack variables $\mathrm{s}_{1}, \mathrm{~s}_{2}, \mathrm{~s}_{3}$ for converting the last three inequalities to equations one forms the problem's first SIMPLEX matrix, which corresponds to the feasible solution $\mathrm{f}(0,0)=0$, as follows:

$$
\left[\begin{array}{ccccc|c}
\mathrm{x}_{1} & \mathrm{x}_{2} & \mathrm{~s}_{1} & \mathrm{~s}_{2} & \mathrm{~s}_{3} & \text { Const. } \\
- & - & - & - & - & - \\
2.5 & 1 & 1 & 0 & 0 & - \\
3 & 3 & 0 & 1 & 0 & 20=\mathrm{s}_{1} \\
1 & 2 & 0 & 0 & 1 & 16=\mathrm{s}_{2} \\
- & - & - & - & - & - \\
-3 & -4 & 0 & 0 & 0 & 0=\mathrm{s}(0,0)
\end{array}\right]
$$

Denote by $\mathrm{L}_{1}, \mathrm{~L}_{2}, \mathrm{~L}_{3}, \mathrm{~L}_{4}$ the rows of the above matrix, the fourth one being the net evaluation row. Since -4 is the smaller (negative) number of the net evaluation row and $\frac{16}{2}<\frac{30}{3}<\frac{20}{1}$, the pivot element 2 lies in the intersection of the third row and second column Therefore, applying the linear transformations $\mathrm{L}_{3} \rightarrow$

${ }^{1}$ The profit is changing depending upon the price of the wood, the salaries of the workers, etc.

${ }^{2}$ The mathematical formulation of the problem using TFNs is not unique. Here we have taken $b=\frac{a+c}{2}$ for all the TFNs involved, but this is not compulsory. The change of the values of the above TFNs, changes of course the ordinary LP problem obtained by ranking them, but the change of its optimal solution is relatively small. 
$\frac{1}{2} \mathrm{~L}_{3}=\mathrm{L}^{\prime}{ }_{3}$ and $\mathrm{L}_{1} \rightarrow \mathrm{L}_{1}-\mathrm{L}^{\prime}{ }_{3}, \mathrm{~L}_{2} \rightarrow \mathrm{L}_{2}-3 \mathrm{~L}^{\prime}{ }_{3}, \mathrm{~L}_{4} \rightarrow \mathrm{L}_{4}+4 \mathrm{~L}^{\prime}{ }_{3}$, one obtains the second SIMPLEX matrix, which corresponds to the feasible solution $\mathrm{f}(0,8)=32$ and it is the following:

$$
\left[\begin{array}{ccccccc}
\mathrm{x}_{1} & \mathrm{x}_{2} & \mathrm{~s}_{1} & \mathrm{~s}_{2} & \mathrm{~s}_{3} & \text { Const. } \\
- & - & - & - & - & - & - \\
2 & 0 & 1 & 0 & -\frac{1}{2} & 12=\mathrm{s}_{1} \\
\frac{3}{2} & 0 & 0 & 1 & -\frac{3}{2} & 6=\mathrm{s}_{2} \\
\frac{1}{2} & 1 & 0 & 0 & \frac{1}{2} & 8=\mathrm{x}_{2} \\
- & - & - & - & - & - \\
-1 & 0 & 0 & 0 & 0 & 32=\mathrm{f}(0,8)
\end{array}\right]
$$

In this matrix the pivot element $3 / 2$ lies in the intersection of the second row and first column, therefore working as above one obtains the third SIMPLEX matrix, which is:

$$
\left[\begin{array}{ccccccc}
\mathrm{x}_{1} & \mathrm{x}_{2} & \mathrm{~s}_{1} & \mathrm{~s}_{2} & \mathrm{~s}_{3} & \mid & \text { Const. } \\
- & - & - & - & - & - & - \\
0 & 0 & 1 & -\frac{4}{3} & -\frac{3}{2} & \mid & 4=\mathrm{s}_{1} \\
1 & 0 & 0 & \frac{2}{3} & -1 & \mid & 4=\mathrm{x}_{1} \\
0 & 1 & 0 & -\frac{1}{3} & 1 & \mid & 6=\mathrm{x}_{2} \\
- & - & - & - & - & - \\
0 & 0 & 0 & \frac{2}{3} & 1 & \mid & 36=\mathrm{f}(4,6)
\end{array}\right]
$$

Since there is no negative index in the net evaluation row, this is the last SIMPLEX matrix. Therefore $\mathrm{f}(4,6)=36$ is the optimal solution maximizing the objective function. Further, since both the decision variables $\mathrm{x}_{1}$ and $\mathrm{x}_{2}$ are basic variables, i.e. they participate in the optimal solution, the above solution is unique.

Converting, by applying Proposition 4, the values of the decision variables in the above solution to TFNs with DoF equal to 1 , one finds that $\mathrm{x}_{1}=(\alpha, 11-2 \alpha, \alpha+1]$ with $\frac{10}{3}<a<\frac{11}{3}$ and $\mathrm{x}_{2}=(a, 17-2 \mathrm{a}, \mathrm{a}+1)$ with. $\frac{16}{3}<\mathrm{a}<\frac{17}{3}$. Therefore a fuzzy expression of the optimal solution states that the factory's maximal profit corresponds to a daily production between $\alpha$ and $\alpha+1$ groups of tables with $3.33<a<3.67$ and between a and a +1 groups of desks with $5.33<\mathrm{a}<5.67$.

However, taking for example $\alpha=3.5$ and $\mathrm{a}=5.5$ and considering the extreme in this case values of the daily construction of 4.5 groups of tables and 6.5 groups of desks, one finds that they are needed 33 in total w.h. for elaboration, whereas the maximum available w.h. are only 30. In other words, a fuzzy expression of the solution does not guarantee that all the values of the decision variables within the boundaries of the corresponding TFNs are feasible solutions.

Example 2: Three kinds of food, say $\mathrm{F}_{1}, \mathrm{~F}_{2}$ and $\mathrm{F}_{3}$, are used in a poultry farm for feeding the chickens, their cost varying between $38-42,17-23$ and $55-65$ cents per kilo respectively. The food $\mathrm{F}_{1}$ contains 1.5 - 2.5 units of iron and $4-6$ units of vitamins per kilo, $\mathrm{F}_{2}$ contains $3.2-4.8,0.6-1.4$ and $\mathrm{F}_{3}$ contains $1.7-$ $2.3,0.8-1.2$ units per kilo respectively. It has been decided that the chickens must receive at least 24 units of iron and 8 units of vitamins per day. How must one mix the three foods so that to minimize the cost of the food? Express the problem's solution with TpFNs of DoF equal to 2.

Solution: Let $\mathrm{x}_{1}, \mathrm{x}_{2}$ and $\mathrm{x}_{3}$ be the quantities in kilos to be mixed for each of the foods $\mathrm{F}_{1}, \mathrm{~F}_{2}$ and $\mathrm{F}_{3}$ 
respectively. Then, using TpFNs the problem's mathematical model could be formulated as follows: ${ }^{3}$ Minimize

subject to the constraints

$$
\mathrm{F}=(38,39,41,42) \mathrm{x}_{1}+(17,18,22,23) \mathrm{x}_{2}+(55,56,64,65] \mathrm{x}_{3}
$$

$$
\begin{gathered}
\mathrm{x}_{1}, \mathrm{x}_{2}, \mathrm{x}_{3} \geq 0,(1.5,1.8,2.2,2.5) \mathrm{x}_{1}+(3.2,3.5,4.5,4.8) \mathrm{x}_{2}+(1.7,1.9,2.1,2.3] \mathrm{x}_{3} \geq[22,23,25,26] \\
{[4,4.5,5.5,6] \mathrm{x}_{1}+[0.6,0.8,1.2,1.4] \mathrm{x}_{2}+[0.8,0.9,1.1,1.2] \mathrm{x}_{3} \geq(6,7,9,10) .}
\end{gathered}
$$

The ranking of the TpFNs leads to the following LP minimization problem of canonical form: Minimize

subject to the constraints

$$
\mathrm{f}\left(\mathrm{x}_{1}, \mathrm{x}_{2}, \mathrm{x}_{2}\right)=40 \mathrm{x}_{1}+20 \mathrm{x}_{2}+60 \mathrm{x}_{3}
$$

The dual of the above problem is the following: Maximize

$$
\mathrm{x}_{1}, \mathrm{x}_{2}, \mathrm{x}_{3} \geq 0,2 \mathrm{x}_{1}+4 \mathrm{x}_{2}+2 \mathrm{x}_{3} \geq 24,5 \mathrm{x}_{1}+\mathrm{x}_{2}+\mathrm{x}_{3} \geq 8
$$

subject to the constraints

$$
\mathrm{g}\left(\mathrm{z}_{1}, \mathrm{z}_{2}\right)=24 \mathrm{z}_{1}+8 \mathrm{z}_{2}
$$

$$
\mathrm{z}_{1}, \mathrm{z}_{2} \geq 0,2 \mathrm{z}_{1}+5 \mathrm{z}_{2} \leq 40,4 \mathrm{z}_{1}+\mathrm{z}_{2} \leq 20,2 \mathrm{z}_{1}+\mathrm{z}_{2} \leq 60
$$

Working similarly with Example 1 it is straightforward to check that the last SIMPLEX matrix of the dual problem is the following:

$$
\left[\begin{array}{ccccccc}
\mathrm{z}_{1} & \mathrm{z}_{2} & \mathrm{~s}_{1} & \mathrm{~s}_{2} & \mathrm{~s}_{3} & \text { Const. } \\
- & - & - & - & - & - & - \\
0 & 1 & \frac{2}{9} & \frac{1}{9} & 0 & \frac{20}{3}=\mathrm{z}_{2} \\
1 & 0 & -\frac{1}{18} & \frac{5}{18} & 0 & \frac{10}{3}=\mathrm{z}_{1} \\
0 & 0 & -\frac{1}{9} & -\frac{4}{9} & 1 & \frac{140}{3}=\mathrm{s}_{3} \\
- & - & - & - & - & - \\
0 & 0 & \frac{4}{9} & \frac{52}{9} & 0 & \frac{400}{3}=g\left(\frac{10}{3}, \frac{20}{3}\right)
\end{array}\right]
$$

Therefore the solution of the original minimization problem is $\mathrm{f}_{\min }=\mathrm{f}\left(\frac{4}{9}, \frac{52}{9}, 0\right)=\frac{400}{3}$.

In other words, the minimal cost of the chickens' food is $\frac{400}{3} \approx 133$ cents and will be succeeded by mixing $\frac{4}{9} \approx 0.44$ kilos from food $\mathrm{F}_{1}$ and $\frac{52}{9} \approx 5.77$ kilos from food $\mathrm{F}_{2}$.

Converting the values of the decision variables in the above solution to TpFNs with DoF equal to 2 one finds by Proposition 5 that $\mathrm{x}_{1}, \mathrm{x}_{2}, \mathrm{x}_{3}$ must be of the form

with

$$
(\alpha, b, c, \alpha+2)
$$

$$
\alpha<b \leq c<\alpha+2, b+c=\frac{18 \mathrm{R}-4 a-4}{7} .
$$

and

$$
\mathrm{R}=\frac{4}{9} \text { or } \mathrm{R}=\frac{52}{9} \text { or } \mathrm{R}=0 \text { respectively. }
$$

For $\mathrm{R}=\frac{4}{9}$ one finds that

$$
b+c=\frac{4-4 a}{7} .
$$

\footnotetext{
${ }^{3}$ The problem's mathematical formulation using TpFNs is not unique, but the change of its optimal solution is relatively small.
} 
Therefore

which gives that

$$
b<\frac{4-4 a}{7}-b \text { or } b<\frac{2-2 a}{7}
$$

$$
\alpha<\frac{2-2 a}{7} \text { or } \alpha<\frac{2}{9} \text {. }
$$

Taking for example $\alpha=\frac{1}{9}$, we find that

$$
b<\frac{2-\frac{2}{9}}{7}=\frac{16}{63} \text {. }
$$

Therefore, taking for example $b=\frac{15}{63}$, we obtain that

Therefore

$$
c=\frac{4-\frac{4}{9}}{7}-\frac{15}{63}=\frac{17}{63} .
$$

$$
\mathrm{x}_{1}=\left(\frac{7}{63}, \frac{15}{63}, \frac{17}{63}, \frac{133}{63}\right)
$$

Working similarly for $\mathrm{R}=\frac{52}{9}$ and $\mathrm{R}=0$ one could obtain

and

$$
\mathrm{x}_{2}=\left(\frac{196}{63}, \frac{340}{63}, \frac{362}{63}, \frac{488}{63}\right)
$$

$$
\mathrm{X}_{3}=\left(-\frac{21}{63},-\frac{15}{63},-\frac{9}{63}, \frac{60}{63}\right)
$$

respectively.

Therefore, since a $\operatorname{TpFN}(\mathrm{a}, \mathrm{b}, \mathrm{c}, \mathrm{d})$ expresses mathematically the fuzzy statement that the interval [b, c] lies within the interval [a,d], a fuzzy expression of the problem's optimal solution states that the minimal cost of the chickens' food will be succeeded by mixing between $\frac{15}{63} \approx 0.24, \frac{17}{63} \approx 0.27$, between $\frac{340}{63} \approx 5.4, \frac{362}{63} \approx 5.75$ and between $-\frac{15}{63} \approx-0.24,-\frac{17}{63} \approx-0.27$ kilos from each one of the foods $\mathrm{F}_{1}$, $\mathrm{F}_{2}$ and $\mathrm{F}_{3}$ respectively. The values of $\mathrm{x}_{3}$ are not feasible and must be replaced by 0 , whereas the values of $\mathrm{x}_{1}$ and $\mathrm{x}_{2}$ must be checked as we did in Example 1 .

Example 3: A cheese-making company produces three different types of cheese $\mathrm{T}_{1}, \mathrm{~T}_{2}$ and $\mathrm{T}_{3}$ by mixing cow-milk (C), sheep-milk (S) and milk powder (P). The required quantities in kilos from each kind of milk for producing a barrel of each of the three types of cheese are depicted, in the form of TFNs, in the following Table:

Table 1. Required quantities of milk ${ }^{4}$

\begin{tabular}{cccc}
\hline & $\mathrm{T}_{1}$ & $\mathrm{~T}_{2}$ & $\mathrm{~T}_{3}$ \\
\hline $\mathrm{C}$ & $(1,2,3)$ & $(5,6,7)$ & $(0.5,1,1.5]$ \\
$\mathrm{S}$ & $(3,4,5)$ & $(2,3,4)$ & $(1.5,2,2.5)$ \\
$\mathrm{P}$ & $(1.8,2,2.2)$ & $(0.7,1,1.3)$ & $(0.8,1,1.2]$ \\
\hline
\end{tabular}

\footnotetext{
${ }^{4}$ The fuzzy data of Table 1 show that the production of a barrel of $\mathrm{T}_{1}$ requires quantities of cow-milk between 1 and 3 kilos of sheep-milk 3 and 5 kilos and of milk powder between 1.8 and 2.2 kilos, etc.
} 
The cheese-maker's profit from the sale of a barrel of cheese is 3 thousand euros for $\mathrm{T}_{1}, 2$ thousand euros for $\mathrm{T}_{3}$, whereas from the sale of a barrel of $\mathrm{T}_{2}$, the production of which becomes necessary for marketing reasons, there is a loss of 1 thousand euros.

At the end of a certain day the stock of the cow-milk is high, so that at least 200 kilos of it must be used the next day, whereas the stock of the sheep-milk is 150 kilos. Further, there exists a stock of 100 kilos of expiring milk powder all of which must be spent the next day. Under the above conditions find with DoF equal to 0.2 which must be the next day's production of cheese in order to maximize the profit from its sale.

Solution: Let $\mathrm{x}_{1}, \mathrm{x}_{2}$ and $\mathrm{x}_{3}$ be the barrels of cheese to be produced of the types $\mathrm{T}_{1}, \mathrm{~T}_{2}$ and $\mathrm{T}_{3}$ respectively. Then the problem is mathematically formulated as follows:

Maximize

subject to the constraints $\mathrm{x}_{1}, \mathrm{x}_{2}, \mathrm{x}_{3} \geq 0$ and

$$
\mathrm{F}=3 \mathrm{x}_{1}-\mathrm{x}_{2}+2 \mathrm{x}_{3}
$$

$$
\begin{gathered}
(1,2,3) \mathrm{x}_{1}+(5,6,7) \mathrm{x}_{2}+(0.5,1,1.5) \mathrm{x}_{3} \geq(199,200,201) \\
(3,4,5) \mathrm{x}_{1}+(2,3,4) \mathrm{x}_{2}+(1.5,2,2.5) \mathrm{x}_{3} \leq(149,150,151) \\
(0.8,1,1.2) \mathrm{x}_{1}+(0.7,1,1.3) \mathrm{x}_{2}+(0.8,1,1.2) \mathrm{x}_{3}=(99,100,101)
\end{gathered}
$$

The ranking of the TFNs leads to the following LP maximization problem of general form ${ }^{5}$ : Maximize

subject to the constraints $\mathrm{x}_{1}, \mathrm{x}_{2}, \mathrm{x}_{3} \geq 0$ and

$$
\mathrm{f}\left(\mathrm{x}_{1}, \mathrm{x}_{2}, \mathrm{x}_{2}\right)=3 \mathrm{x}_{1}-\mathrm{x}_{2}+2 \mathrm{x}_{3}
$$

$$
\begin{gathered}
2 \mathrm{x}_{1}+6 \mathrm{x}_{2}+\mathrm{x}_{3} \geq 200 \\
4 \mathrm{x}_{1}+3 \mathrm{x}_{2}+2 \mathrm{x}_{3} \leq 150 \\
2 \mathrm{x}_{1}+\mathrm{x}_{2}+\mathrm{x}_{3}=100
\end{gathered}
$$

Adding the surplus variable $\mathrm{s}_{1}$ to the first inequality, the slack variable $\mathrm{s}_{2}$ to the second one and the artificial variables $t_{1}$ and $t_{2}$ to the first inequality and the last equation one turns all the special constraints to equations. Next, adding by members the two equations containing the artificial variables, one forms the problem's first generalized SIMPLEX matrix as follows:

$$
\left[\begin{array}{ccccccccc}
\mathrm{x}_{1} & \mathrm{x}_{2} & \mathrm{x}_{3} & \mathrm{~s}_{1} & \mathrm{~s}_{2} & \mathrm{t}_{1} & \mathrm{t}_{2} & \text { Const. } \\
- & - & - & - & - & - & - & - & - \\
2 & 6 & 1 & -1 & 0 & 1 & 0 & 200=\mathrm{t}_{1} \\
4 & 3 & 2 & 0 & 1 & 0 & 0 & 150=\mathrm{s}_{2} \\
2 & 1 & 3 & 0 & 0 & 0 & 1 & 100=\mathrm{t}_{2} \\
- & - & - & - & - & - & - & - & - \\
-3 & 1 & -2 & 0 & 0 & 0 & 0 & 0=\mathrm{f}(0,0,0) \\
- & - & - & - & - & - & - & - & - \\
4 & 7 & 4 & -1 & 0 & 1 & 1 & \mathrm{t}_{1}+\mathrm{t}_{2}=300
\end{array}\right]
$$

The rows of the artificial variables $t_{1}$ and $t_{2}$ are the, so called, anonymous rows of the above matrix. For the pivoting process, considering all the columns containing at least one positive number in the anonymous rows, we choose the one having the greatest positive number in the last row (of $t_{1}+t_{2}$ ), i.e. the column of $\mathrm{x}_{2}$. Then, since $\frac{200}{6}<\frac{150}{3}<\frac{100}{1}$, the pivot element 6 lies in the first row. Therefore, applying the proper linear transformations among the rows of the matrix one forms the second generalized SIMPLEX matrix as follows:

\footnotetext{
${ }^{5}$ It is recalled that a LP problem of general form differs from a problem of canonical form to the fact that there exists at least a constraint having the inverse sign of inequality (here $\geq$ ), whereas there could also exist constraints with the sign of equality.
} 


$$
\left[\begin{array}{ccccccccc}
\mathrm{x}_{1} & \mathrm{x}_{2} & \mathrm{x}_{3} & \mathrm{~s}_{1} & \mathrm{~s}_{2} & \mathrm{t}_{1} & \mathrm{t}_{2} & \text { Const. } \\
- & - & - & - & - & - & - & - & - \\
\frac{1}{3} & 1 & \frac{1}{6} & -\frac{1}{6} & 0 & \frac{1}{6} & 0 & \frac{100}{3}=\mathrm{x}_{2} \\
3 & 0 & \frac{3}{2} & \frac{1}{2} & 1 & -\frac{1}{2} & 0 & 50=\mathrm{s}_{2} \\
\frac{5}{3} & 0 & \frac{17}{6} & \frac{1}{6} & 0 & \frac{1}{6} & 1 & \frac{200}{3}=\mathrm{t}_{2} \\
- & - & - & - & - & - & - & - \\
-\frac{10}{3} & 0 & -\frac{13}{6} & \frac{1}{6} & 0 & -\frac{1}{6} & 0 & -\frac{100}{3}=\mathrm{f}\left(0, \frac{100}{3}, 0\right) \\
- & - & - & - & - & - & - & - \\
\frac{5}{3} & 0 & \frac{17}{6} & \frac{1}{6} & 0 & -\frac{1}{6} & 1 & \frac{200}{3}=\mathrm{t}_{1}+\mathrm{t}_{2}
\end{array}\right]
$$

The pivot element $17 / 6$ lies now in the intersection of the column of $x_{3}$ and the row of $t_{2}$ and the third generalized SIMPLEX matrix is the following:

$$
\left[\begin{array}{ccccccccc}
\mathrm{x}_{1} & \mathrm{x}_{2} & \mathrm{x}_{3} & \mathrm{~s}_{1} & \mathrm{~s}_{2} & \mathrm{t}_{1} & \mathrm{t}_{2} & \text { Const. } \\
- & - & - & - & - & - & - & - & - \\
\frac{4}{17} & 1 & 0 & \frac{3}{17} & 0 & \frac{3}{17} & -\frac{1}{17} & \frac{500}{17}=\mathrm{x}_{2} \\
\frac{36}{17} & 0 & 0 & \frac{7}{17} & 1 & -\frac{7}{17} & -\frac{9}{17} & \frac{250}{17}=\mathrm{s}_{2} \\
\frac{10}{17} & 0 & 1 & \frac{1}{17} & 0 & -\frac{1}{17} & -\frac{6}{17} & \frac{400}{17}=\mathrm{x}_{3} \\
- & - & - & - & - & - & - & - & - \\
\frac{35}{17} & 0 & 0 & \frac{5}{17} & 0 & \frac{5}{17} & \frac{13}{17} & \frac{300}{17}=\mathrm{f}\left(0, \frac{500}{17}, \frac{400}{17}\right) \\
- & - & - & - & - & - & - & - & - \\
0 & 0 & 0 & 0 & 0 & 0 & 0 & 0=\mathrm{t}_{1}+\mathrm{t}_{2}
\end{array}\right]
$$

Therefore, omitting the last row and the columns of the artificial variables one obtains the problem's first canonical SIMPLEX matrix.

Next, continuing the process in the standard way one finally reaches the optimal solution

$$
\mathrm{f}_{\max }=\mathrm{f}\left(\frac{125}{18}, \frac{250}{9}, \frac{175}{9}\right)=\frac{575}{18} .
$$

Converting the values $\mathrm{x}_{1}=\frac{125}{18} \approx 6.94, \mathrm{x}_{2}=\frac{250}{9} \approx 27.78, \mathrm{x}_{3}=\frac{175}{9} \approx 19.44$ to TFNs with DoF equal to 0.2 one finds by Proposition 5 that $\mathrm{x}_{1} \approx(\alpha, 20.63-2 \alpha, \alpha+0.2)$, with $6.81<\alpha<6.87, \mathrm{x}_{2} \approx(a, 83.13-2 a$, $a+0.2)$, with $27.64<a<27.71$ and $\mathrm{x}_{3} \approx(\mathrm{a}, 58.13-2 \mathrm{a}, \mathrm{a}+0.2)$, with $19.31<\mathrm{a}<19.37$.

Taking for example $\alpha=6.85, a=27.7$ and $\mathrm{a}=19.35$ one finds that $\mathrm{x}_{1} \approx(6.85,6.93,7.05), \mathrm{x}_{2} \approx(27.7$, $27.99,28.19)$, and $\mathrm{x}_{3} \approx(19.35,19.43,19.55)$, which are very close to the values of the decision variables in the crisp optimal solution. In general, the smaller is the chosen DoF of the FNs involved in the problem's optimal solution, the more creditable its corresponding fuzzy expression.

\section{Conclusion}

A new technique was developed in this paper for solving Fuzzy LP problems by ranking the FNs involved in their statements and by solving the ordinary LP problem obtained in this way with the standard theory. Real-life examples were also presented to illustrate our method. In LP problems with a vague structure a fuzzy expression of their solution is often preferable than the crisp one. This was attempted in the present 
work by converting the values of the decision variables in the optimal solution of the corresponding ordinary LP problem to FNs with the desired DoF. The smaller is the value of the chosen DoF, the more creditable the fuzzy expression of the problem's optimal solution.

An analogous method could be applied for solving Grey LP problems and systems of equations with fuzzy or grey data and this is the main target of our future research on the subject.

\section{References}

1. Dantzig, G.B., "Maximization of a linear function of variables subject to linear inequalities", 1947. Published in: T.C. Koopmans (Ed.): Activity Analysis of Production and Allocation, pp. 339-347. Wiley \& Chapman - Hall, New York, London, 1951.

2. Dantzig, G.B., Reminiscences about the origins of linear programming, Technical Report of Systems Optimization Laboratory 81-5, Department of Operations Research, Stanford University, CA, April 1981.

3. Taha, H.A., Operations Research - An Introduction, Second Edition, Collier Macmillan, N.Y., London, 1967.

4. Tanaka H., .Asai K., "Fuzzy linear programming problems with fuzzy numbers", Fuzzy Sets and Systems, 13, 1-10, 1984.

5. Verdegay, J.L., "A dual approach to solve the fuzzy linear programming problem", Fuzzy Sets and Systems, 14, 131-141, 1984.

6. Stephen Dinagar, D., Kamalanathan, S., "Solving Linear Programming Problem Using New Ranking Procedures of Fuzzy Numbers", International Journal of Applications of Fuzzy Sets and Artificial Intelligence, 281-292, 2017.

7. Huang, G., Dan Moore, R., "Grey linear programming, its solving approach, and its application", International Journal of Systems Science, 24(1), 159-172, 1993

8. Seyed H., Razavi H., Akrami, H., Hashemi, S.S., "A multi objective programming approach to solve grey linear programming", Grey Systems: Theory and Application, 2(2), 259-271, 2012.

9. Zadeh, L.A., "Fuzzy sets", Information and Control, 8 (1965), 338-353.

10. Klir,. G.J.\& Folger, T.A., Fuzzy Sets, Uncertainty and Information, Prentice-Hall, London, 1988.

11. Kaufmann, A. \& Gupta, M., Introduction to Fuzzy Arithmetic, Van Nostrand Reinhold Company, New York, 1991.

12. Dubois, D., Prade, H., Fuzzy Sets and Systems: Theory and Applications, Academic Press, New York, 1980.

13. van Broekhoven, E. \& De Baets, B., "Fast and accurate centre of gravity defuzzification of fuzzy system outputs defined on trapezoidal fuzzy partitions", Fuzzy Sets and Systems, 157(7), 904-918, 2006.

14. Voskoglou, M. Gr., Finite Markov Chain and Fuzzy Logic Assessment Models: Emerging Research and Opportunities, Createspace.com. - Amazon, Columbia, SC, 2017.

15. Wang, M.L., Wang, H. F., Lung, L..C., "Ranking Fuzzy Number Based on Lexicographic Screening Procedure", International Journal of Information Technology and Decision Making, 4, 663-678, 2005.

16. Wang, Y.J., Lee, H.S., "The Revised Method of Ranking Fuzzy Numbers with an Area Between the Centroid and Original Points", Computers and Mathematics with Applications, 55(9), 2033-2042, 2008. 